\title{
Tendencias de investigación en enfermería
}

\author{
Trends in Nursing Research \\ Tendências de pesquisa em enfermagem
}

DOI: 10.5294/aqui.2014.14.3.1

Me siento muy honrada con la invitación para escribir este editorial sobre las tendencias en la investigación en enfermería para la Revista Aquichan. Mis opiniones se centran en la evolución de los temas que las enfermeras deciden estudiar, la influencia de la financiación sobre los temas que se consideran importantes para el estudio y el peso de las posiciones editoriales sobre la publicación de informes de investigación en enfermería.

La historia de la investigación en enfermería debe ser considerada dentro del contexto de la sociedad en la que la investigación se lleva a cabo (1). Dadas las similitudes en el contexto social de la enfermería alrededor del mundo - típicamente considerado como un arte y apenas recientemente también como una ciencia, de bajo estatus y servil a la medicina y, últimamente, solo como socio colaborativo en otros campos relacionados con el estudio de la salud; con una distribución de género predominantemente femenina que solo recientemente incluye un mayor porcentaje de varones; con un enfoque de aprendizaje hacia la educación en enfermería que hace relativamente poco tiempo evolucionó hacia un enfoque profesional; y siempre con pocos recursos para llevar a cabo la investigación-, se desprende que la investigación en enfermería ha progresado de una manera similar en la mayoría de todos los países, si no en todos $(1,2)$.

\section{La evolución de los temas para la investigación en enfermería}

La investigación en enfermería se inició en la década de 1850 con Florence Nightingale, cuya perspectiva de la enfermería se centró en la interacción entre la persona y su entorno $(2,3)$, y se institucionalizó en la medida en que la educación en enfermería evolucionó desde programas de diplomado en hospitales, hasta la enseñanza universitaria, que incluía programas de doctorado, a partir de la década de los veinte.

Las enfermeras siempre han estado interesadas en la realización de investigaciones sobre la atención al paciente. Sin embargo, la mayoría de los temas de investigación desde los años veinte hasta finales de los sesenta se centraron en la educación en enfermería y en las enfermeras (3). Las facultades de enfermería querían saber cómo facilitar el aprendizaje de los estudiantes en cuanto a las habilidades necesarias para la práctica de la enfermería, y los administradores de la disciplina querían saber cómo pasaban su tiempo y qué hacían las enfermeras cuando se dedicaban a la práctica de la profesión. La investigación al respecto fue denominada "estudios de tiempo y movimiento". La investigación sobre la educación en enfermería continúa hoy en día, pero en menor medida que en el pasado. Por ejemplo, un tema importante en la investigación contemporánea al respecto es la eficacia de la simulación con el fin de que los estudiantes puedan aprender las habilidades de la práctica de enfermería. Los estudios sobre enfermeras también continúan hoy en día, pero de igual manera en menor grado que en el pasado. Por ejemplo, los típicos temas contemporáneos son las experiencias de las enfermeras en la atención de pacientes con determinados problemas de salud, como cáncer 0 enfermedades cardiacas, respiratorias o infecciosas.

En la década de los setenta hubo una recomposición de los principales temas de investigación en enfermería, desde la educación y las enfermeras hacia las experiencias relacionadas con la 
salud de las personas, las familias y las comunidades. Este enfoque sustantivo de la investigación en enfermería, que representa lo que siempre ha estado implícito pero ahora es una búsqueda más explícita de evidencia para la práctica de la enfermería ha evolucionado de una manera similar en todo el mundo. Hoy en día el objetivo final explícito de la investigación en enfermería es encontrar pruebas que se puedan utilizar en la práctica (3).

En cuanto a la investigación en enfermería, el énfasis contemporáneo en la práctica basada en la evidencia ha evolucionado a partir de la utilización de la investigación, que fue el enfoque de muchas publicaciones desde la década de los setenta hasta finales de los noventa. La utilización de la investigación se refiere a "las medidas preparatorias necesarias para las acciones relacionadas con la investigación que — cuando son implementadas y se sostienen- Ilevan a [la práctica basada en la evidencia]" (4). La utilización de la investigación se ha convertido recientemente en investigación traslacional, la cual "es un proceso de investigación utilizado para determinar qué condiciones, costos y recursos se necesitan para pasar de la generación y la comprobación de la teoría a la utilización de la misma o a la práctica basada en la evidencia" (5).

Aunque se ha avanzado mucho desde los tiempos de Nightingale en cuanto a la cantidad de investigación en enfermería y a la diversidad de los temas estudiados, una gran parte de esa investigación se ha llevado a cabo sin una base conceptual o teórica explícita. Poco a poco las enfermeras están comenzando a reconocer que toda la investigación se guía por un modelo conceptual (6), se lleva a cabo para desarrollar teorías, los hallazgos de la investigación son teorías, y las teorías son la evidencia para la práctica (5). Yo sostengo que toda la investigación en enfermería tendrá mucho más valor si es guiada por un modelo conceptual de la disciplina (7), en vez de que el modelo conceptual que guía la investigación continúe siendo implícito. Por ejemplo, he explicado cómo mis colegas, estudiantes de posgrado y yo realizamos un programa de investigación guiado explícitamente por el modelo de adaptación de Roy (8) y otro programa de investigación orientado explícitamente por la teoría de Rogers (9) sobre seres humanos unitarios (10).

\section{La influencia de la financiación en la investigación en enfermería}

La Sociedad de Honor de Enfermería, Sigma Theta Tau, fue la primera organización en Estados Unidos que proveyó fondos para la investigación en enfermería en 1936. Hoy en día, esta organización internacional y sus subdivisiones locales proporcionan financiación para la investigación en enfermería llevada a cabo por sus miembros en muchos países.

En Estados Unidos, el Gobierno federal comenzó a financiar la investigación en enfermería en la década de los cuarenta; la mayoría de los fondos federales ahora provienen de los Institutos Nacionales de Salud. Las fundaciones privadas comenzaron a financiar la investigación en enfermería en Estados Unidos en la década de los cincuenta, y los filántropos comenzaron su financiación en la década de los sesenta. Una revisión de las revistas de enfermería que contienen los informes de las investigaciones llevadas a cabo por las enfermeras en todo el mundo indica que cierto grado de financiamiento gubernamental y algún tipo de financiamiento otorgado por organizaciones profesionales están disponibles en varios países.

La financiación de la investigación en enfermería en cada país depende de la economía general del mismo, especialmente en los que se encuentran en vía de desarrollo (11). Sin embargo, como Parahoo (2) señaló: "No es prudente generalizar en cuanto a los países en desarrollo, ya que varían de acuerdo con sus niveles de desarrollo económico. En algunos de esos países la investigación de enfermería es más desarrollada que en los demás", a pesar de la economía general.

Las prioridades de investigación establecidas por las agencias gubernamentales, las organizaciones, las fundaciones privadas y los filántropos han dado forma al tipo y a los temas de los estudios realizados por las enfermeras. La investigación experimental diseñada para probar las intervenciones innovadoras en enfermería ha sido una prioridad constante, incluso cuando aún no se comprenden las descripciones y las explicaciones de las intervenciones experimentales. Es probable que el énfasis en la investigación experimental sobre la disciplina refleje la preocupación tanto de las enfermeras como de los financiadores por la práctica basada en la evidencia dirigida a la mejora de la calidad de vida de las personas que requieren cuidados de enfermería. Sin embargo, la tendencia a comenzar con la experimentación resulta en las Ilamadas "cajas negras" del conocimiento; es decir, el desarrollo del conocimiento de los efectos de una intervención sin entender el contenido detallado de la misma y por qué ella es eficaz 0 ineficaz (12). El enfoque de la caja negra 
se centra solo en si la intervención fue eficaz en el cumplimiento de los resultados esperados, pero no tiene en cuenta las posibles variables exógenas al cumplimiento de estos resultados. En consecuencia, el enfoque de la caja negra puede resultar en una intervención que cumple con los resultados esperados en un ensayo clínico en particular, pero carece de la fiabilidad y validez para seguir cumpliendo con los resultados a través del tiempo o para otras muestras de la misma población (13).

Por tanto, no se sabe cuál intervención es eficaz; cuándo, dónde y para quién, y por qué y cómo la intervención es eficaz (14). Cuando se lleva a cabo una investigación de caja negra los hallazgos no constituyen teorías bien desarrolladas las cuales son la mejor prueba de la práctica, sino que proporcionan evidencia para la práctica basada en creencias clínicas obstinadas, opiniones de las autoridades y el sentido común $(5,15)$.

Es posible evitar la investigación estilo "caja negra" si las enfermeras están dispuestas a llevar a cabo programas de investigación que comiencen con estudios cualitativos y descriptivos diseñados para comprender las experiencias de la gente en cuanto a condiciones relacionadas con la salud, para luego avanzar hacia los estudios de correlación de las relaciones entre las experiencias relacionadas con la salud y los factores que influyen en esas experiencias, y solo así pasar a los estudios experimentales de las pruebas de los efectos de las intervenciones sobre los resultados que reflejen una mejora en las experiencias relacionadas con la salud.

Otra fuente de financiación para la investigación en enfermería son las universidades que ofrecen becas para profesores y estudiantes de posgrado. La financiación proporcionada por las universidades tiende a aumentar a medida que la institución amplía su misión para incluir no solamente la docencia sino también la investigación. Por ejemplo, la Universidad de Massachusetts en Boston ha evolucionado desde una misión casi exclusivamente centrada en la enseñanza al momento de su fundación en 1964, hasta el punto en que se considera a sí misma como una "excelente universidad pública dedicada a la investigación y con alma para la enseñanza" (Declaración de la Visión de la Universidad de Massachusetts en Boston, 2010). Algunas universidades establecen las prioridades generales para la investigación en relación directa con la misión y los objetivos de la universidad. Por ejemplo, la mencionada Universidad ofrece financiación para investigaciones a cargo de la facultad y los estudiantes de posgrado que aborden sus metas en cuanto a la mayor participación de la comunidad y la colaboración internacional.

\section{La publicación de investigaciones en enfermería}

La difusión de la investigación en enfermería se produce a través de conferencias internacionales, nacionales, regionales y locales en muchos países. Sin embargo, las presentaciones en las conferencias solo llegan a audiencias relativamente pequeñas. Los artículos de revistas, por el contrario, tienen el potencial de llegar a cientos o incluso miles de lectores en todo el mundo a través de material impreso y la Internet. Por tanto, es imperativo que las enfermeras que realizan investigación transfieran rápidamente sus presentaciones a artículos que luego sean enviados a las revistas con el fin de ser considerados para publicación. Los editores de revistas y los miembros del Comité Editorial pueden animar a la presentación de dichos artículos cuando asistan a conferencias y cuando interactúen con colegas de su institución de origen.

También es imprescindible identificar explícitamente en las publicaciones el modelo conceptual que orienta la investigación y la teoría que se genera o que se encuentra bajo prueba. La inclusión de modelos y teorías conceptuales explícitos en los informes de investigación sería mucho más habitual de lo que se observa en la actualidad si los editores de revistas y los revisores son receptivos a tales informes de investigación y les dan apoyo. Esto se convertirá en la norma cuando los editores de revistas de enfermería exijan que los investigadores expliquen clara e integralmente toda la estructura conceptual-teórica-empírica de sus estudios (5).

La gran mayoría de las revistas de enfermería en todo el mundo incluyen informes de las investigaciones realizadas por las enfermeras en muchos países. Por ejemplo, la International Journal of Nursing Studies, el Journal of Advanced Nursing, el Journal of Nursing Scholarship y la Revista Aquichan publican trabajos desarrollados por enfermeras investigadoras de varios países. Los estudios publicados en estas y muchas otras revistas son excelentes ejemplos de las "muchas historias [que hay que contar] de diferentes personas en distintos momentos con diversas visiones sobre los tipos de 'buena' ciencia, 'técnicas válidas' y conocimiento 'valioso' necesarios para atender a los pacientes, [y] avanzar la disciplina" (1).

La transición de informes de investigación de un solo autor a informes escritos por equipos de investigadores se está haciendo evidente en las revistas de enfermería. Este cambio refleja la cre- 
ciente complejidad de los temas estudiados y el énfasis contemporáneo en los diseños de investigación de métodos mixtos que requieren equipos de investigadores, algunos de los cuales son expertos en diseños cualitativos, otros son expertos en diseños cuantitativos, y otros más lo son en la integración de los datos cualitativos y cuantitativos (16). Preveo que la colaboración entre equipos de investigadores en enfermería al interior de y entre las universidades y los organismos clínicos dentro de un país y entre países aumentará a medida que avancemos en nuestra búsqueda de teorías empíricamente adecuadas que sirvan de evidencia para la práctica de la enfermería (5).

Aunque la creación y gestión de equipos de investigadores para la realización y el informe de la investigación son un reto, la riqueza y la profundidad de este tipo de investigación colaborativa pueden superan los desafíos. Parahoo (2) hizo una advertencia acerca de las colaboraciones entre países que presenta un reto particularmente intrigante. Explicó que los proyectos conjuntos de investigación entre las enfermeras de los países más ricos y las de los más pobres pueden ser beneficiosos o pueden ser un obstáculo. "Los proyectos en conjunto implican la transferencia de habilidades y oportunidades útiles de aprendizaje. Sin embargo, pueden dar lugar a cambios en la imagen de las sociedades occidentales" (2) que impidan la igualdad de las relaciones entre los investigadores de enfermería de los países más ricos y de los más pobres. Este desafío puede superarse solo si las enfermeras colaboradoras que realizan investigación entienden y respetan la cultura de cada país, incluyendo el estilo de vida, el lenguaje y las percepciones sobre la investigación y los investigadores.

Aunque la necesidad de tener equipos de investigadores es reconocida y alentada por las universidades y los organismos clínicos, no es fácil evaluar de manera justa la contribución particular al estudio y al informe de cada miembro del equipo de investigación. Los editores de revistas pueden evitar la existencia de este desafío al exigir una declaración de las contribuciones específicas de cada investigador al estudio y al informe de investigación.

Para terminar, quiero dar las gracias a María Elisa Moreno Fergusson, editora de la Revista Aquichan, y a los miembros del Consejo de Redacción de la revista por la oportunidad de compartir con los lectores mis pensamientos acerca de las tendencias en la investigación en enfermería.

Jacqueline Fawcett, RN, PhD, ScD (Hon), FAAN

Profesora del Departamento de Enfermería Universidad de Massachusetts, Boston (EE.UU.). jacqueline.fawcett@umb.edu

\section{Referencias}

1. D'Antonio P. Toward a history of research in nursing. Nursing Research. 1997;46:105-110.

2. Parahoo K. Nursing research: Principles, process, and issues. $2^{\text {nd }}$ ed. New York: Palgrave Macmillan; 2006.

3. Grove SK, Burns N, Gray JR. The practice of nursing research: Appraisal, synthesis, and generation of evidence. $7^{\text {th }}$ ed. St. Louis: Elsevier/Saunders; 2013.

4. Stetler CB. Updating the Stetler model of research utilization to facilitate evidence-based practice. Nursing Outlook. 2001b;49:272-279.

5. Fawcett J, Garity J. Evaluating research for evidence-based nursing research. Philadelphia: F. A. Davis; 2009.

6. Popper KR. Conjectures and refutations: The growth of scientific knowledge. New York: Harper and Row; 1965.

7. Fawcett J. The added value of nursing conceptual model-based research. Journal of Advanced Nursing. 2008;61:583.

8. Roy C. The Roy adaptation model. 3rd ed. Upper Saddle River, NJ: Pearson; 2009.

9. Rogers ME. Nursing science and the space age. Nursing Science Quarterly. 1992;5:27-34.

10. Clarke PN, Fawcett J. Life as a nurse researcher. Nursing Science Quarterly. 2014;27:37-41.

11. Tierney AJ. Organization report: The development of nursing research in Europe. European Nurse. 1997;2:73-81. 
12. Conn VS. Unpacking the black box: Countering the problem of inadequate intervention descriptions in research reports. Western Journal of Nursing Research. 2012;34:427-433.

13. Wallace Kazer M, Bailey DE, Whittemore R. Out of the black box: Expansion of a theory-based intervention to selfmanage the uncertainty associated with active surveillance (AS) for prostate cancer. Research and Theory for Nursing Practice. 2010;24:101-112.

14. Sidani S, Braden CJ. Evaluating nursing interventions: A theory-driven approach. Thousand Oaks: Sage; 1998.

15. Pomeroy VM, Niven DS, Barrow S, Faragher EB, Tallis RC. Unpacking the black box of nursing and therapy practice for post-stroke shoulder pain: A precursor to evaluation. Clinical Rehabilitation. 2001;15:67-63.

16. Creswell JW. A concise introduction to mixed methods research. Los Angeles: Sage; 2015. 